\title{
STABLE PROCESSES WITH AN ABSORBING BARRIER
}

\author{
BY \\ DANIEL RAY( ${ }^{(1)}$
}

1. Introduction. Let $\{X(t), t \geqq 0\}$ be a symmetric stable process on the real line, with exponent $\alpha, 0<\alpha<2$, and with the initial value $X(0)=0$. We may assume [3] that with proper normalization the paths $X(\cdot)$ are right continuous functions of $t \geqq 0$, bounded on finite $t$-intervals.

For such a process, if $I=(a, b)$ is an arbitrary (finite or infinite) open interval containing the origin, the random variable

$$
T=T(X)=\inf \{t \geqq 0 \mid X(t) \notin I\},
$$

that is, the time of first passage from the interval $I$, is measurable on the sample space of the process. So likewise is the place of first passage, $X(T)$.

The process in question is known [5] to satisfy an extended Markov property:

$$
Y(t)=X(T+t)-X(T), \quad t \geqq 0,
$$

defines a process equivalent to the original one and independent of the values $\{X(t), 0 \leqq t \leqq T\}$ of the original process up to the first passage time.

Using the extended Markov property and a method of analytic continuation, we will derive some properties of the joint distribution of $T$ and $X(T)$. One explicit formula will appear: for $a=-\infty$, the distribution of the place $X(T)$ of first passage from the origin across the point $b>0$ has the density

$$
\begin{aligned}
\frac{d}{d x} \operatorname{Pr}\{X(T) \leqq x\} & =\frac{\sin \pi \alpha / 2}{\pi} \frac{1}{x}\left(\frac{b}{x-b}\right)^{\alpha / 2}, & & x \geqq b, \\
& =0, & & x<b .
\end{aligned}
$$

In particular, for $0<\alpha<2$, the first passage occurs continuously with probability zero.

An equivalent formula can be given for the stable process with an absorbing barrier at $b$ : the derivative

$$
P_{0}(x, t)=\frac{d}{d x} \operatorname{Pr}\{X(t) \leqq x ; T>t\}
$$

Received by the editors December 5, 1956.

(1) Research under contract with the Office of Naval Research. 
exists, and

$$
\begin{aligned}
\int_{0}^{\infty} P_{0}(x, t) d t & =\frac{1}{(\Gamma(\alpha / 2))^{2}} \int_{0}^{\min (b, b-x)} d \xi \xi^{\alpha / 2-1}(\xi+|x|)^{\alpha / 2-1}, & & x \leqq b, \\
& =0, & & x>b .
\end{aligned}
$$

Although explicit formulas have been found for the distribution of $T$ in the case $a=-\infty[1,2]$ and for $\int_{0}^{\infty} P_{0}(x, t) d t$ in the case $\alpha=1, a, b$ finite [6], our method does not yield them. However, some information can be derived concerning the resolvent of the absorbing barrier process in the general case. Similar properties to those which follow have been proved recently by Joanne Elliott [4] by analytic methods.

NOTE ADDED IN PROOF: The combinatorial lemma which forms the basis of [1] and [2] has recently been proved by methods similar to those of this paper. An equivalent proof, by J. G. Wendel, will appear in the Bulletin of the American Mathematical Society.

For $(a, b)$ an arbitrary open interval containing the origin, but not the entire real line, let $P_{0}(x, t)$ be defined as above. The resolvent $\int_{0}^{\infty} e^{-\lambda t} P_{0}(x, t) d t$, $\lambda \geqq 0$, vanishes for $x$ outside $(a, b)$, and is continuous at the boundaries $a$ and $b$; in fact, as $x$ decreases to $a$,

$$
\begin{aligned}
\int_{0}^{\infty} e^{-\lambda t} P_{0}(x, t) d t & =O\left((x-a)^{\alpha / 2}\right), & & a>-\infty, \lambda \geqq 0, \\
& =O\left(|x|^{-(1+\alpha)}\right), & & a=-\infty, \lambda>0, \\
& =O\left(|x|^{-(1-\alpha / 2)}\right), & & a=-\infty, \lambda=0,
\end{aligned}
$$

with a similar behavior at $b$. The resolvent is the sum of a singular part and a regular part:

$$
\begin{aligned}
& \int_{0}^{\infty} e^{-\lambda t} P_{0}(x, t) d t=S_{\lambda}(|x|)-\overline{R_{\lambda}}(x-a)-\overline{R_{\lambda}^{+}}(b-x), \\
& \lambda \geqq 0, a<x<b .
\end{aligned}
$$

The singular part, independent of $a$ and $b$, is the resolvent of the original process, if $\lambda>0$, or its finite part, if $\lambda=0$ :

$$
\begin{aligned}
S_{\lambda}(\xi) & =\frac{1}{\pi} \int_{0}^{\infty} \frac{\cos \xi z}{\lambda+z^{\alpha}} d z, & \lambda>0, \\
& =(2 \cos \pi \alpha / 2 \Gamma(\alpha))^{-1} \xi^{\alpha-1}, & \lambda=0, \alpha \neq 1, \\
& =\frac{1}{\pi} \log \frac{1}{\xi}, & \lambda=0, \alpha=1 .
\end{aligned}
$$

The regular part $R_{\lambda}(\xi)=R_{\lambda}^{ \pm}(\xi)$ depends on $a$ and $b$ as well as $\lambda$ as parameters, 
and vanishes if the corresponding boundary is infinite. $S_{\lambda}(\xi)$ and $R_{\lambda}(\xi)$ are each infinitely differentiable for $0<\xi<\infty$. If $\lambda>0, S_{\lambda}(\xi)$ and $R_{\lambda}(\xi)$ are completely monotonic: that is,

$$
\begin{array}{ll}
(-1)^{n} \frac{d^{n}}{d \xi^{n}} S_{\lambda}(\xi) \geqq 0, & \lambda>0, n=0,1,2, \cdots, \\
(-1)^{n} \frac{d^{n}}{d \xi^{n}} R_{\lambda}(\xi) \geqq 0, & \lambda>0, n=0,1,2, \cdots,
\end{array}
$$

while if $\lambda=0,-(d / d \xi) S_{\lambda}(\xi)$ and $-(d / d \xi) R_{\lambda}(\xi)$ are completely monotonic: the above equations hold for $n=1,2, \cdots$.

2. The Désiré-André equation. The basis of our study is the form of the extended Markov property which is sometimes called the Désiré-André equation. Denote by $I$ the open interval $(a, b)$, by $I^{\prime}$ its complement, and by $p(x, t)$ the transition density for the stable process,

$$
p(x, t)=\frac{1}{\pi} \int_{0}^{\infty} d z \cos z x e^{-t z^{\alpha}} .
$$

Denote again by $P_{0}(x, t)$ the derivative of the distribution function

$$
\operatorname{Pr}\{X(t) \leqq x, T>t\},
$$

the existence of which follows from the fact that

$$
\begin{aligned}
\operatorname{Pr}\left\{x<X(t)<x^{\prime}, T>t\right\} & \leqq \operatorname{Pr}\left\{x<X(t)<x^{\prime}\right\} \\
& =\int_{x}^{x^{\prime}} p(\xi, t) d \xi .
\end{aligned}
$$

Then the extended Markov property immediately implies the Désiré-André equation

(6) $p(x, t)=P_{0}(x, t)+\int_{I^{\prime}} \int_{0}^{t} p\left(x-y, t-t^{\prime}\right) \operatorname{Pr}\left\{T \in d t^{\prime}, X(t) \in d y\right\}$.

We note for future reference that because of $(6) P_{0}(x, t)$ is for each $t>0$ a continuous function of $x$; hence in particular $P_{0}(a, t)=P_{0}(b, t)=0$.

For $\lambda>0, z$ real, define

$$
\begin{aligned}
\phi(\lambda, z) & =\int_{0}^{\infty} e^{-\lambda t} \int_{I^{\prime}} e^{i z x} \operatorname{Pr}\{T \in d t, X(t) \in d x\} \\
& =E\left\{e^{-\lambda T} e^{i z X(T)}\right\} \\
\psi(\lambda, z) & =\int_{0}^{\infty} d t \int_{I} d x e^{-\lambda t} e^{i z x} P_{0}(x, t) .
\end{aligned}
$$


Then upon taking the Laplace transform with respect to $t$ and the Fourier transform wth respect to $x$ of equation (6),

$$
\frac{1}{\lambda+|z|^{\alpha}}=\psi(\lambda, z)+\frac{1}{\lambda+|z|^{\alpha}} \phi(\lambda, z),
$$

for $\lambda>0, z$ real.

3. The single absorbing barrier. Specializing to the case $a=-\infty, b>0$, we can make use of the fact that since $I=(-\infty, b), I^{\prime}=[b, \infty)$, the integrals defining $\phi(\lambda, t)$ and $\psi(\lambda, t)$ for fixed $\lambda>0$ converge for $\operatorname{Im} z \geqq 0, \operatorname{Im} z \leqq 0$, respectively, and that $e^{-i z b} \phi(\lambda, z)$ and $e^{-i z b} \psi(\lambda, z)$ are bounded analytic functions in the upper and lower half planes, respectively. Set

$$
\Phi(z)=e^{-i z b} z^{-\alpha / 2} \phi(\lambda, z)
$$

for $0 \leqq \arg z \leqq \pi . \Phi$ is analytic in the upper half plane and because of ( 7$), \Phi$ has the analytic continuation

$$
\Phi(z)=e^{-i z b} z^{-\alpha / 2}\left(1-\left(\lambda+z^{\alpha}\right) \psi(\lambda, z)\right)
$$

for $-\pi \leqq \arg z \leqq 0$. We have, again using (7),

$$
\begin{aligned}
\Phi\left(r e^{i \pi}\right) & =e^{i r b} e^{-i \pi \alpha / 2} r^{-\alpha / 2} \phi(\lambda,-r) \\
& =e^{i r b} e^{-i \pi \alpha / 2} r^{-\alpha / 2}\left(1-\left(\lambda+r^{\alpha}\right) \psi(\lambda,-r)\right), \\
\Phi\left(r e^{-i \pi}\right) & =e^{i r b} e^{i \pi \alpha / 2} r^{-\alpha / 2}\left(1-\left(\lambda+e^{-i \pi \alpha} r^{\alpha}\right) \psi(\lambda,-r)\right) \\
& =e^{i r b} e^{i \pi \alpha / 2} r^{-\alpha / 2} \frac{1-\left(\lambda+e^{-i \pi \alpha} r^{\alpha}\right)}{1-\left(\lambda+r^{\alpha}\right)} \phi(\lambda,-r) .
\end{aligned}
$$

If $\alpha=2$, then

$$
\Phi\left(r e^{i \pi}\right)=\Phi\left(r e^{-i \pi}\right):
$$

it follows that $e^{-i z b} \phi(\lambda, z)$ is a bounded entire function, hence a constant:

$$
\begin{aligned}
& \phi(\lambda, z)=c_{\lambda} e^{i z b}, \\
& \psi(\lambda, z)=\frac{1-c_{\lambda} e^{i z b}}{+z^{2}} .
\end{aligned}
$$

Since $\psi(\lambda, z)$ is analytic in the lower half plane,

$$
c_{\lambda}=e^{-(\lambda)^{1 / 2} b},
$$

yielding the well known formula for the first passage time distribution for Brownian motion.

When $0<\alpha<2, \Phi(z)$ is bounded for $-\pi \leqq \arg z \leqq \pi,|z| \geqq \delta>0$, and tends to zero as $z$ becomes infinite along the rays $\arg z= \pm \pi$. Hence by the Phrag- 
mén-Lindelöf Theorem [7], $\Phi(z)$ tends to zero as $z$ becomes infinite, uniformly for $|\arg z| \leqq \pi$. By a similar argument, $z^{\alpha / 2} \Phi(z)$ is bounded for $|z| \leqq \delta$, $|\arg z| \leqq \pi$.

We next apply the Cauchy integral formula to $\Phi$, integrating $(w-z)^{-1} \Phi(w)$, for fixed $z>0$, about a contour consisting of the circles $|z|=\delta,|z|=R$, connected by line segments along arg $w=\pi$ and arg $w=-\pi$. Because of the properties of $\Phi$ given in the last paragraph, the contributions to the Cauchy integral formula from the circular parts of the contour vanish as $R$ becomes infinite, and $\delta$ tends to zero. In the limit, the formula becomes

$$
\begin{aligned}
\Phi(z) & =e^{-i z b} z^{-\alpha / 2} \phi(\lambda, z) \\
& =\frac{1}{2 \pi i} \int_{0}^{\infty} \frac{d r}{r+z}\left(\Phi\left(r e^{-i \pi}\right)-\Phi\left(r e^{i \pi}\right)\right) \\
& =\frac{\sin \pi \alpha / 2}{\pi} \int_{0}^{\infty} \frac{d r}{r+z} r^{-\alpha / 2} e^{i r b}(1-\lambda \psi(\lambda,-r)),
\end{aligned}
$$

or,

$$
\phi(\lambda, z)=\frac{\sin \pi \alpha / 2}{\pi} \int_{b}^{\infty} \frac{d r}{r+1} r^{-\alpha / 2} e^{i(r+1) z b}(1-\lambda \psi(\lambda,-r z)) .
$$

Now as $\lambda$ decreases to zero, $\phi(\lambda, t)$ increases to

$$
\phi(0, z)=E\left\{e^{i z X(T)}\right\}
$$

remaining bounded in absolute value by one. Likewise, ( 7 ) implies that $\lambda \psi(\lambda,-r)$ tends boundedly to zero, whereas $r^{-\alpha / 2}(r+1)^{-1}$ is integrable on $(0, \infty)$. Thus (8) becomes

$$
\begin{aligned}
\phi(0, z) & =\frac{\sin \pi \alpha / 2}{\pi} \int_{0}^{\infty} \frac{d r}{r+1} r^{-\alpha / 2} e^{i(r+1) z b} \\
& =\frac{\sin \pi \alpha / 2}{\pi} \int_{b}^{\infty} \frac{d x}{x}\left(\frac{b}{x-b}\right)^{\alpha / 2} e^{i x z} .
\end{aligned}
$$

This equation and the uniqueness of the Fourier-Stieltjes transform imply the result (1).

Turning to the result (2), we use (6) and the remark after (6) to write

$$
\begin{aligned}
P_{0}(x, t) & =P_{0}(x, t)-P_{0}(b, t) \\
& =p(x, t)-p(b, t) \\
- & \int_{b}^{\infty} \int_{0}^{t} \operatorname{Pr}\left\{T \in d t^{\prime}, X(T) \in d y\right\}\left(p\left(x-y, t-t^{\prime}\right)-p\left(b-y, t-t^{\prime}\right)\right) .
\end{aligned}
$$

Now when neither $y$ nor $y^{\prime}$ are zero, $p(y, t)-p\left(y^{\prime}, t\right)$ is integrable with respect 
to $t$, and in fact,

$$
\begin{aligned}
\int_{0}^{\infty}\left(p(y, t)-p\left(y^{\prime}, t\right)\right) d t & =\frac{1}{\pi} \int_{0}^{\infty} \frac{\cos y z-\cos y^{\prime} z}{z^{\alpha}} d z \\
& =\frac{\sin \pi \alpha / 2}{\pi} \int_{0}^{\infty}\left(e^{-|y| w}-e^{-\left|y^{\prime}\right| w}\right) \frac{d w}{w^{\alpha}} .
\end{aligned}
$$

Thus for $x<b, x \neq 0$, the above and (1) imply

$$
\begin{aligned}
& \int_{0}^{\infty} P_{0}(x, t) d t=\frac{\sin \pi \alpha / 2}{\pi} \int_{0}^{\infty}\left(e^{-|x| w}-e^{-b w}\right) \frac{d w}{w^{\alpha}} \\
& \quad+\left(\frac{\sin \pi \alpha / 2}{\pi}\right)^{2} \int_{b}^{\infty} \frac{d y}{y}\left(\frac{b}{y-b}\right)^{\alpha / 2} \int_{0}^{\infty} \frac{d w}{w^{\alpha}}\left(e^{-(y-b) w}-e^{-(y-x) w}\right) \\
& =\left(\frac{\sin \pi \alpha / 2}{\pi}\right)^{2} \int_{0}^{\infty} \frac{d y}{y+1} y^{-\alpha / 2} \int_{0}^{\infty} \frac{d w}{w^{\alpha}}\left(e^{-|x| w}-e^{-b w}+e^{-b y w}-e^{-(b y+b-x) w}\right) \\
& =\left(\frac{\sin \pi \alpha / 2}{\pi}\right)^{2} \int_{0}^{\infty} \int_{0}^{\infty} \frac{d y d w}{y+w}(y w)^{-\alpha / 2}\left(e^{-|x| w}-e^{-b(y+w)+x w}\right) \\
& =\left(\frac{\sin \pi \alpha / 2}{\pi}\right)^{2} \int_{0}^{\infty} \int_{0}^{\infty} \frac{d y d w}{y+w}(y w)^{-\alpha / 2}\left(e^{-|x| w}-e^{-b(y+w)+x y}\right) .
\end{aligned}
$$

But

$$
\begin{aligned}
e^{-|x| w} e^{-\min (b, b-x)(y+w)} & =e^{-b(y+w)+x w}, & & x<0, \\
& =e^{-b(y+w)+x y}, & & x>0 .
\end{aligned}
$$

Hence in either case

$$
\begin{aligned}
\int_{0}^{\infty} P_{0}(x, t) d t \\
=\left(\frac{\sin \pi \alpha / 2}{\pi}\right)^{2} \int_{0}^{\infty} \int_{0}^{\infty} \frac{d y d w}{y+w}(y w)^{-\alpha / 2} e^{-|x| w}\left(1-e^{-(y+w) \min (b, b-x)}\right) \\
=\left(\frac{\sin \pi \alpha / 2}{\pi}\right)^{2} \int_{0}^{\infty} d y \int_{0}^{\infty} d w(y w)^{-\alpha / 2} e^{-|x| w} \int_{0}^{\min (b, b-x)} e^{-(y+w) \xi} d \xi \\
=\left(\frac{\sin \pi \alpha / 2}{\pi}\right)^{2}(\Gamma(1-\alpha / 2))^{2} \int_{0}^{\min (b, b-x)} d \xi \xi^{-(1-\alpha / 2)}(\xi+|x|)^{-(1-\alpha / 2)},
\end{aligned}
$$

which is just the result (2).

4. The resolvent in the general case. If $a$ and $b$ are finite, then $\psi(\lambda, z)$ is entire and $\phi(\lambda, z)=\phi_{r}(\lambda, z)+\phi_{l}(\lambda, z)$, with $\phi_{r}$ and $\phi_{l}$ analytic in the upper and lower half planes, respectively. The analysis of the preceding section leads to an equation similar to (8), but yields no explicit formulas. An ap- 
proach based on (6) appears more fruitful in deriving general properties of the resolvent of the absorbing barrier process.

We assume, as we may without loss of generality, that $b<\infty$. Then as in the preceding section

$$
\begin{aligned}
\int_{0}^{\infty} P_{0}(x, t) d t= & \int_{0}^{\infty} P_{0}(x, t) d t-\int_{0}^{\infty} P_{0}(b, t) d t \\
= & \frac{\sin \pi \alpha / 2}{\pi} \int_{0}^{\infty}\left(e^{-w|x|}-e^{-w b}\right) \frac{d w}{w^{\alpha}} \\
& -\frac{\sin \pi \alpha / 2}{\pi} \int_{b}^{\infty} \operatorname{Pr}\{X(T) \in d y\} \int_{0}^{\infty}\left(e^{-w(y-x)}-e^{-w(y-b)}\right) \frac{d w}{w^{\alpha}} \\
& -\frac{\sin \pi \alpha / 2}{\pi} \int_{-\infty}^{a} \operatorname{Pr}\{X(T) \in d y\} \int_{0}^{\infty}\left(e^{-w(x-y)}-e^{-w(b-y)}\right) \frac{d w}{w^{\alpha}} .
\end{aligned}
$$

But by (5), if $y$ and $y^{\prime}$ are positive,

$$
\begin{aligned}
\frac{\sin \pi \alpha / 2}{\pi} \int_{0}^{\infty}\left(e^{-w y}-e^{-w y^{\prime}}\right) \frac{d w}{w^{\alpha}} & =\frac{\sin \pi \alpha / 2}{\pi} \Gamma(2-\alpha) \int_{y}^{y^{\prime}} \frac{d \xi}{\xi^{2-\alpha}} \\
& =S_{0}(y)-S_{0}\left(y^{\prime}\right) .
\end{aligned}
$$

Hence, with reference to (4),

$$
-\frac{d}{d \xi} R_{0}^{+}(\xi)=\frac{\sin \pi \alpha / 2}{\pi} \Gamma(2-\alpha) \int_{b}^{\infty} \operatorname{Pr}\{X(T) \in d y\}(\xi+y-b)^{-(2-\alpha)} ;
$$

it follows that $-d R_{0}^{+}(\xi) / d \xi$ is completely monotonic for $0<\xi<\infty$, as are also $-d R_{0}^{-}(\xi) / d \xi$ and $-d S_{0}(\xi) / d \xi$.

For $\lambda>0$, taking the Laplace transform of (6),

$$
\begin{aligned}
\int_{0}^{\infty} e^{-\lambda t} P_{0}(x, t) d t= & \frac{1}{\pi} \int_{0}^{\infty} \frac{\cos x z}{\lambda+z^{\alpha}} d z \\
& -\int_{b}^{\infty} E\left\{e^{-\lambda T} ; X(T) \in d y\right\} \frac{1}{\pi} \int_{0}^{\infty} \frac{\cos (x-y) z}{\lambda+z^{\alpha}} d z \\
& -\int_{-\infty}^{a} E\left\{e^{-\lambda T} ; X(T) \in d y\right\} \frac{1}{\pi} \int_{0}^{\infty} \frac{\cos (x-y) z}{\lambda+z^{\alpha}} d z
\end{aligned}
$$

where $E\left\{e^{-\lambda T} ; X(T) \in d y\right\}$ is the measure

$$
E\left\{e^{-\lambda T} ; X(T) \in d y\right\}=\int_{0}^{\infty} e^{-\lambda t} \operatorname{Pr}\{T \in d t ; X(T) \in d y\} .
$$

Now it is easy to show that for $\xi>0, \lambda>0$, 


$$
S_{\lambda}(\xi)=\frac{1}{\pi} \int_{0}^{\infty} \frac{\cos \xi z}{\lambda+z^{\alpha}} d z=\frac{\sin \pi \alpha / 2}{\pi} \int_{0}^{\infty} e^{-\xi w} \frac{w^{\alpha} d w}{\lambda^{2}+2 \lambda w^{\alpha} \cos \pi \alpha / 2+w^{2 \alpha}} ;
$$

from this and (4) it follows that

$$
\begin{aligned}
R_{\lambda}^{+}(\xi)= & \frac{\sin \pi \alpha / 2}{\pi} \int_{0}^{\infty} e^{-\xi w} \frac{w^{\alpha} d w}{\lambda^{2}+2 \lambda w^{\alpha} \cos \pi \alpha / 2+w^{2 \alpha}} \\
& \cdot \int_{b}^{\infty} e^{-(y-b) w} E\left\{e^{-\lambda T} ; X(T) \in d y\right\} .
\end{aligned}
$$

The last two equations imply that for $\lambda>0, S_{\lambda}(\xi)$ and $R_{\lambda}^{+}(\xi)$ are completely monotonic for $0<\xi<\infty$. A similar equation and result hold of course for $R_{\lambda}^{-}(\xi)$.

Suppose now that $a=-\infty$; then $R_{\lambda}^{-}(\xi)$ vanishes. By a change of variable and an easily validated interchange of limit and integration,

$$
\begin{aligned}
\lim _{\xi \rightarrow \infty} \xi^{1+\alpha} R_{\lambda}^{+}(\xi)= & \frac{\sin \pi \alpha / 2}{\pi} \lim _{\xi \rightarrow \infty} \int_{0}^{\infty} e^{-w} \frac{w^{\alpha} d w}{\lambda^{2}+2 \lambda w^{\alpha} \xi^{-\alpha} \cos \pi \alpha / 2+w^{2 \alpha} \xi^{-2 \alpha}} \\
& \cdot \int_{b}^{\infty} e^{-(y-b) w / \xi} E\left\{e^{-\lambda T} ; X(T) \in d y\right\} \\
= & \frac{\sin \pi \alpha / 2}{\pi \lambda^{2}} \Gamma(1+\alpha) E\left\{e^{-\lambda T}\right\} .
\end{aligned}
$$

Since, similarly,

$$
\lim _{\xi \rightarrow \infty} \xi^{1+\alpha} S_{\lambda}(\xi)=\frac{\sin \pi \alpha / 2}{\pi \lambda^{2}} \Gamma(1+\alpha),
$$

the second result under (3) has been verified.

If $\lambda=0, a=-\infty$, then because of the explicit expression (2),

$$
\begin{aligned}
\lim _{x \rightarrow-\infty}|x|^{1-\alpha / 2} \int_{0}^{\infty} P_{0}(x, t) d t & =\frac{1}{(\Gamma(\alpha / 2))^{2}} \lim _{x \rightarrow-\infty} \int_{0}^{b} d \xi \xi^{\alpha / 2-1}(1+\xi /|x|)^{\alpha / 2-1} \\
& =\frac{b^{\alpha / 2}}{\Gamma(\alpha / 2) \Gamma(1+\alpha / 2)}
\end{aligned}
$$

yielding the third part of (3).

For $a>-\infty$, the fact that for all $x, x^{\prime}$

$$
\begin{aligned}
\int_{x}^{x^{\prime}} P_{0}(\xi, t) d \xi & =\operatorname{Pr}\left\{x<\xi<x^{\prime} ; a<X(\tau)<b, 0 \leqq \tau \leqq t\right\} \\
& \leqq \operatorname{Pr}\left\{x<\xi<x^{\prime} ; a<X(\tau), 0 \leqq \tau \leqq t\right\}
\end{aligned}
$$


implies, because of (2),

$$
\begin{aligned}
\int_{0}^{\infty} e^{-\lambda t} P_{0}(x, t) d t & \leqq \int_{0}^{\infty} P_{0}(x, t) d t \\
& \leqq \frac{1}{(\Gamma(\alpha / 2))^{2}} \int_{0}^{\min (|a|, x-a)} d \xi \xi \xi^{\alpha / 2-1}(\xi+|x|)^{\alpha / 2-1}
\end{aligned}
$$

for $x>a$. Hence for $\lambda \geqq 0$,

$$
\begin{aligned}
\limsup _{x \rightarrow a}(x-a)^{-\alpha / 2} \int_{0}^{\infty} & e^{-\lambda t} P_{0}(x, t) d t \\
& \leqq \frac{1}{(\Gamma(\alpha / 2))^{2}} \lim _{x \downarrow a}(x-a)^{-\alpha / 2} \int_{0}^{x-a} d \xi \xi^{\alpha / 2-1}(\xi+|x|)^{\alpha / 2-1} \\
& =\frac{|a|^{\alpha / 2-1}}{\Gamma(\alpha / 2) \Gamma(1+\alpha / 2)}
\end{aligned}
$$

which is the first part of (3).

We remark finally, without giving details, that using the methods of this section and Equation (8) and its analogue for $a$ and $b$ finite, one can prove that as $x$ decreases to $a$,

$$
\int_{0}^{\infty} e^{-\lambda t} P_{0}(x, t) d t \sim C_{\lambda}(x-a)^{\alpha / 2}
$$

hence the result (3) gives the correct orders of magnitude in all three cases.

\section{REFERENCES}

1. D. A. Darling, The maximum of sums of stable random variables, Trans. Amer. Math. Soc. vol. 83 (1956) pp. 164-169.

2. M. D. Donsker and G. E. Baxter, On the distribution of the supremum functional for processes with stationary independent increments, Trans. Amer. Math. Soc. vol. 85 (1957) pp. 73-87.

3. J. L. Doob, Stochastic processes, New York, 1953.

4. Joanne Elliott, paper to appear in the Illinois Journal of Mathematics.

5. G. A. Hunt, Some theorems concerning Brownian motion, Trans. Amer. Math. Soc. vol. 81 (1956) pp. 294-319.

6. M. Kac and H. Pollard, On the distribution of the maximum of partial sums of independent random variables, Canadian J. Math. vol. 2 (1950) pp. 375-384.

7. E. C. Titchmarsh, The theory of functions, Oxford, 1939.

Cornell University, ItHACA, N. Y. 